\title{
Descrição morfológica do diafragma do sagui-de-tufo-branco (Callithrix jacchus) $^{1}$
}

\author{
Thais B. Lessa ${ }^{2 *}$, Maria Vitória P. Constantino², Luana Célia S. da Silva ${ }^{2}$, Paulo R.S. Santos ${ }^{2}$, \\ Antônio C. de A. Neto ${ }^{2}$, Maria A. Miglino ${ }^{2}$, Pedro P. Bombonato ${ }^{2}$ e Carlos E. Ambrósio ${ }^{3}$
}

\begin{abstract}
Lessa T.B., Constantino M.V.P., Silva L.C.S., Santos S.R.P., Assis Neto A.C., Miglino M.A, Bombonato P.P. \& Ambrósio C.E. 2012. [Morphological description of diaphragm of white-tufted-ear-marmoset (Callithrix jacchus).] Descrição morfológica do diafragma do sagui-de-tufo-branco (Callithrix jacchus). Pesquisa Veterinária Brasileira 32(6):553-558. Departamento de Cirurgia, Programa de Pós-Graduação em Anatomia dos Animais Domésticos e Silvestres, Faculdade de Medicina Veterinária, Universidade de São Paulo, Av. Prof. Dr. Orlando Marques de Paiva 87, Cidade Universitária, São Paulo, SP 05508 270, Brazil. E-mail: thaisblessa@usp.br

The diaphragm muscle found only in mammals is the main muscle in the respiratory process, serving as the border between the thoracic and abdominal cavities. Its significance also is highlighted in research conducted with grafts using various types of biological membranes for the repair of diaphragmatic defects which may cause diaphragmatic hernias. In spite of many studies already conducted in non-human primates, especially in regard to the new world species Callithrix jacchus (white-tufted-ear-marmoset) from northeastern Brazil, research involving use of the diaphragm in such species is nonexistent. Thus, the objective was to characterize the morphology and biometry of the diaphragm in C. jacchus of both sexes, analyzing possible structural differences between males and females. To this end, we used four adult white-tufted-ear-marmosets from a commercial breeder, two males and two females that had died of natural causes. After fixed in $10 \%$ formaldehyde solution, the animals were dissected for photo documentation, the diaphragm was collected for biometrics (length and width) with a caliper, and for histological processing by hematoxylin-eosin and Trichrome Masson. The measurements showed that there were no significant differences between males and females. The topography and the presence of three openings (foramen vena cava, aortic et esophageal hiatus) in the extension of the diaphragm corroborate descriptions in classical literature for other mammals. Regarding the histological findings, it was concluded that the muscular fibers are arranged in an organized shape, with a large diameter and basal nuclei, and showed similar characteristics of skeletal muscle in males and females.
\end{abstract}

INDEX TERMS: Primate, marmoset, Callithrix jacchus, diaphragm, morphology.

RESUMO.- O músculo diafragma, encontrado apenas nos mamíferos, é o principal músculo no processo respiratório, servindo de fronteira entre as cavidades torácica e abdo-

\footnotetext{
${ }^{1}$ Recebido em 25 de julho de 2011.

Aceito para publicação em 24 fevereiro de 2012

${ }^{2}$ Departamento de Cirurgia, Programa de Pós-Graduação em Anatomia dos Animais Domésticos e Silvestres, Faculdade de Medicina Veterinária (FMVZ), Universidade de São Paulo (USP), Av. Prof. Dr. Orlando Marques de Paiva 87, Cidade Universitária, São Paulo, SP 05508 270, Brasil. *Autor para correspondência: thaisblessa@usp.br

${ }^{3}$ Departamento de Ciências Básicas, Faculdade de Zootecnia e Engenharia de Alimentos (FZEA), USP, São Paulo, SP.
}

minal. Sua importância também ganha destaque em pesquisas realizadas no âmbito dos enxertos, empregando-se diversos tipos de membranas biológicas para o reparo de defeitos diafragmáticos, os quais podem gerar hérnias diafragmáticas. Apesar de muitos estudos já conduzidos para com os primatas não humanos, especialmente no que tange a espécie do novo mundo Callithrix jacchus (Sagui-de-tufo-branco), oriundo do nordeste brasileiro, as pesquisas envolvendo o uso do diafragma em tal espécie é inexistente. Deste modo objetivou-se caracterizar a morfologia e a biometria do diafragma na espécie Callithrix jacchus de ambos os sexos, analisando possíveis divergências estruturais en- 
tre machos e fêmeas. Para tal foram utilizados quatros animais, 2 machos e 2 fêmeas, adultos, que vieram a óbito por causas naturais, provenientes de um criadouro comercial. Após fixação em solução de formaldeído $10 \%$ os animais foram devidamente dissecados para fotodocumentação e em seguida o diafragma coletado para efetuação da biometria (comprimento e largura) com o uso de um paquímetro e para o processamento histológico por meio da coloração de hematoxilina-eosina e tricrômio de masson, da porção muscular. As mensurações feitas permitiram concluir que não houve diferenças significativas entre machos e femeas. A topografia e a presença de três aberturas (forame da veia cava caudal, hiato aórtico e esofágico) na extensão do diafragma corroboram com descrições na literatura classica para outros mamíferos. A presença de um centro tendíneo em "V" difere do encontrado para animais como o peixe-boi e porquinho-da-india, mas é similar ao encontrado para o gambá-de-orelhas-brancas e rato albino. No que diz respeito aos achados histológicos conclui-se que as fibras musculares estão dispostas de forma organizada, apresentam diâmetro grande e núcleos basais, tendo, portanto, características similares do músculo estriado esquelético tanto nos animais machos como nas fêmeas.

TERMOS DE INDEXAÇÃO: Primata, sagui, Callithrix jacchus, diafragma, morfologia.

\section{INTRODUÇÃO}

O diafragma é o músculo mais importante no ato da respiração apresentando o formato de uma cúpula com sua superfície convexa voltada para o abdome e sua superfície côncava voltada para a cavidade torácica. Servindo de fronteira entre a cavidade torácica e abdominal, apenas nos mamíferos, e conseqüentemente se encontra coberto pelo peritônio na face abdominal e estando separada da pleura pela fáscia endotorácica. Durante cada ciclo respiratório o formato da cavidade torácica é alterado tendo em vista que durante a inspiração, processo ativo, o volume de tal cavidade precisa aumentar para permitir a entrada do ar para os pulmões. E esta alteração de formato ocorre especialmente pelo músculo diafragma, em grau menor pelo movimento das articulações costocondrais e entre a cabeça das costelas e as vertebrais torácicas. Desta forma quando ocorre a contração do diafragma a curvatura de sua cúpula é reduzida e sua parte central se movimenta caudalmente tendo como resultado a entrada do ar para a cavidade torácica (Getty 1986, Dyce et al. 1987).

Sua importância também ganha destaque em pesquisas realizadas no âmbito dos enxertos para a reparação de defeitos diafragmáticos que muitas vezes culminam com a formação de hérnias diafragmáticas, estas apresentando alta incidência na medicina veterinária. Muitas são as membranas biológicas utilizadas para o reparo de tais defeitos, estes tanto de origem congênita quanto traumática, quando a aproximação das bordas do diafragma é inviável, tais como o pericárdio, peritônio, fáscia lata e músculos como o grande dorsal, reto e transverso do abdome ou mesmo o próprio diafragma de outra espécie distinta da receptora. Ao se empregar tais materiais para a reconstituição de defeitos dia- fragmáticos busca-se atingir um crescimento de tecido conjuntivo satisfatório, o mínimo de reação inflamatória e um suporte da diferença de pressão quando da respiração de forma adequada. Caracterizando assim uma satisfatória resposta cicatricial com boa consolidação (Filho et al. 2003).

As pesquisas envolvendo o uso do músculo diafragma em primatas não humanos para enxertos são inexistentes, apesar de muitos destes animais, principalmente o sagui-de-tufo-branco (Callithrix jacchus), original do nordeste brasileiro, ser o mais conhecido e estudado do seu gênero de primatas do novo mundo. Historicamente a utilização de tal espécie foi direcionada para pesquisas neurológicas, fisiológicas, reprodutivas, comportamentais e doenças infecciosas. Devido principalmente a sua alta taxa reprodutiva, porte pequeno variando de 350 a $450 \mathrm{~g}$, manejo facilitado, menor custo de manunteção e peculariedades fisiológicas aos humanos. Mas em contrapartida a diversos estudos conduzidos existe ainda uma carência grande em pesquisas básicas em primatas não humanos, dentre elas aquelas que abordam aspectos morfológicos e anatômicos (Manfield 2003).

0 estudo torna-se relevante para subsidiar futuras utilizações do diafragma para o reparo de defeitos seja em espécies animais ou mesmo para humanos. Bem como também para a obtenção de informações de cunho morfológico que possam fundamentar discussões do ponto de vista funcional e fisiológico de tais animais.

Diante do exposto o objetivo deste estudo envolveu a caracterização morfológica e biométrica do diafragma do sagui-de-tufo-branco (Callithrix jacchus) de ambos os sexos, analisando as possíveis divergências estruturais entre macho e fêmea.

\section{MATERIAL E MÉTODOS}

Foram utilizados quatro espécimes de Callithrix jacchus (Sagui-de-tufo-branco), adultos, 2 fêmeas e 2 machos, provenientes de um criadouro comercial localizado em Atibaia/SP, e que vieram a óbito por causas naturais, aprovado pelo Comitê de Bioética da Universidade de São Paulo no 1995/2010. Tais animais foram encaminhados à Faculdade de Medicina Veterinária e Zootecnia da Universidade de São Paulo (USP) para estudos anatômicos. Os animais foram fixados em formaldeído $10 \%$ por um tempo mínimo de 72 horas, para então sua posterior dissecação. Denominou-se de N1 para o macho de 3 anos, N2 para o macho de 5 anos, N3 para a fêmea de 3 anos e de N4 para a fêmea de 8 anos.

Após a fixação procedeu-se com uma incisão e rebatimento em sentido dorsal da pele na linha mediana ventral, do manúbrio do osso esterno até a cicatriz umbilical, com posterior retirada da fáscia subcutânea e grupos musculares. Permitindo assim acesso ao músculo diafragma, tanto pela sua face torácica quanto pela abdominal. Realizou-se então a fotodocumentação de tal músculo. E em seguida procedeu-se com a sua retirada efetuando-se uma incisão ao longo das porções lombares, costais e esternais para assim ocorrer a preservação de seu formato anatômico. Neste momento então procedeu-se com a coleta dos dados morfométricos os quais foram obtidos com o uso de um paquímetro. Para a mensuração tanto do comprimento quanto da largura do músculo diafragma foi tomado como base dois pontos equidistantes do mesmo Toda nomenclatura adotada no estudo foi baseada na Nomenclatura Anatômica Veterinária.

Em ambos os animais analisados efetuou-se a coleta de fragmentos das porções musculares os quais foram fixados em para- 
formaldeido a $4 \%$ durante um período superior a 24 horas. Com posterior desidratação em séries crescentes de etanol (70-100\%), diafanizados em xilol e inclusão em Paraplast $®$ (Leica/Germany). Permitindo assim a confecção de blocos retangulares com base de $3 \times 4 \mathrm{~cm}$. Em seguida foram realizados cortes de $5 \mathrm{~mm}$ em micrótomo (Leica RM 2065) para a obtenção das lâminas as quais foram posteriormente desparafinizadas em estufa a $60^{\circ} \mathrm{C}$ por 2 horas. Após tal procedimento as lâminas foram coradas com $\mathrm{He}$ matoxilina-Eosina (HE) para análise da estrutura e das características histológicas do músculo, e com a coloração de Tricrômio de Masson com o objetivo de visualização das fibras colágenas. As lâminas foram então fotomicrografadas em Microscópio Olympus BX 60 acoplado a câmera Axio CAM HRc, utilizando-se o software Zeissâ KS 400.

\section{RESULTADOS}

Após a dissecação observou-se que o músculo diafragma do sagui-de-tufo-branco possuía formato de cúpula tendo concavidade para o tórax e convexidade ao abdome. A face torácica estava separada da pleura pela fascia endotorácica e a face abdominal estava separada do peritônio pela fascia transversal. Apresentou-se caudalmente ao pulmão e coração, estando fixo a este ultimo por meio do ligamento frenicopericárdico (Fig.1A-B). E apresentava-se cranialmente ao fígado possuindo dois ligamentos a tal órgão, o coronário e o falciforme.

Com relação a suas estruturas notou-se que havia uma parte central de tecido conjuntivo fibroso denominado de centro tendíneo, onde existia uma abertura para a passagem da veia cava caudal, denominado de forame da veia cava caudal. 0 músculo diafragma possuía também uma parte carnosa periférica chamada de porção muscular onde ocorria a presença de duas aberturas, a primeira denominada de Hiato esofágico, para a parte abdominal do esôfago, e a segunda chamada de hiato aórtico, para a passagem da aorta, veia ázigos e ducto torácico (Fig.2).

Pôde-se notar ainda que o músculo diafragma possuía três divisões na porção muscular. A primeira correspondia à parte esternal, fixada na parte cranial do processo xifói-

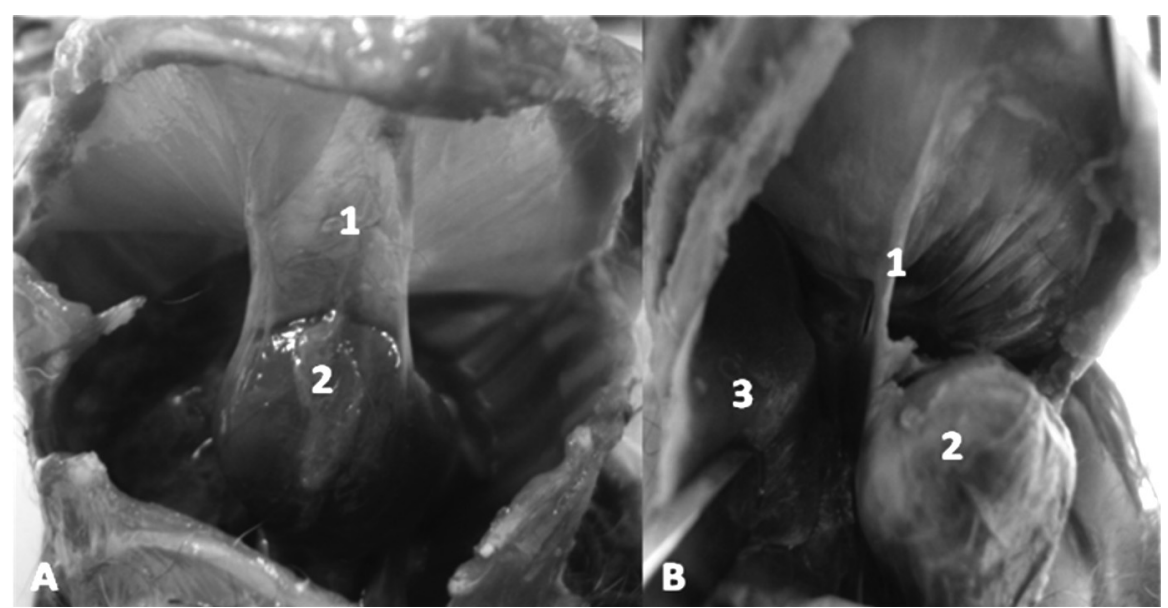

Fig.1. Face torácica do músculo diafragma do sagui-de-tufo-branco (Callithrix jacchus). (A) Ligamento frenicopericárdico (1) e pericárdio envolvendo o coração (2). (B) Ligamento frenicopericárido (1), coração (2) e lobo pulmonar esquerdo (3).

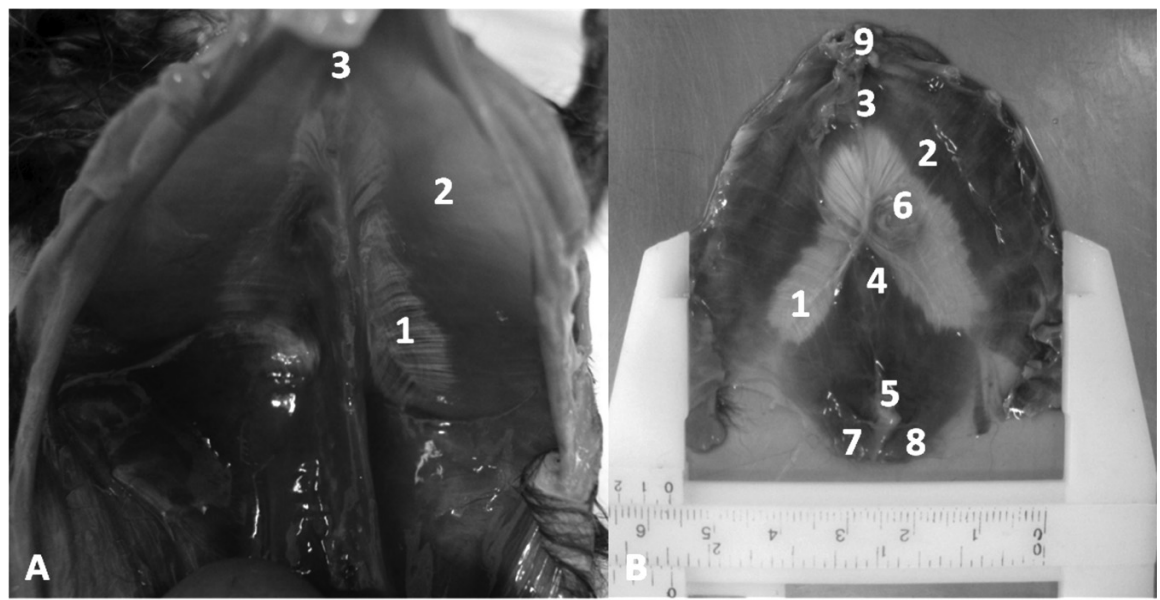

Fig.2. Face abdominal do músculo diafragma do sagui-de-tufo-branco (Callithrix jacchus). (A) Centro tendíneo (1), parte costal (2) e parte esternal (3) da cúpula diafragmática. (B) Centro tendíneo (1), a parte costal (2) e parte esternal (3) da cupula diafragmática, o hiato esofágico (4), hiato aórtico (5), forame da veia cava caudal (6), pilar diafragmático direito (7) e esquerdo (8) e o processo xifóide (9). 
de do esterno. A segunda representada pela parte costal, cujos feixes se ligavam às cartilagens costais e às costelas correspondentes. E a terceira correspondia aos pilares diafragmáticos, direito e esquerdo, que seguiam em direção ao centro tendíneo. Apresentava também tendões periféricos que se ligavam ventralmente ao processo xifóide do osso esterno, dorsalmente às três primeiras vértebras lombares, lateralmente às costelas $\left(7^{\mathrm{a}}, 8^{\underline{a}}, 9^{\mathrm{a}} \mathrm{a}\right.$ e $10^{\underline{a}}$ e ápices da $11^{\mathrm{a}}$, $12^{\underline{a}}$ e $13^{a}$ ) e às cartilagens costais correspondentes. Destes tendões periféricos emergiam feixes musculares que seguiam radialmente para unir-se ao centro tendíneo.

As medidas biométricas do comprimento e da largura do músculo diafragma na espécie Callithrix jacchus, bem como os valores da media e desvio padrão, estão representados nos Quadros 1 e 2. Salienta-se entre machos e femeas o desvio padrao do comprimento foi de $0,249 \mathrm{~cm}$ e para a largura foi de $0,17 \mathrm{~cm}$.

Na microscopia óptica das lâminas analisadas observou-se que a fibra muscular esquelética do músculo diafragma apresentava-se com estriações transversais, devido à alternância de faixas escuras e claras (Fig.3A-B). Estas estriações se apresentam devido à repetição de unidades, denominadas de sarcômeros (Junqueira 2004). A fibra muscular possuía miofibrilas, que se encontravam paralelas ao
Quadro 1. Valores biométricos do músculo diafragma para animais machos da espécie Sagui-de-tufo-branco (Callithrix jacchus)

\begin{tabular}{ccc}
\hline Animal & Comprimento & Largura \\
\hline N1 (macho) & $5,812 \mathrm{~cm}$ & $5,600 \mathrm{~cm}$ \\
N2 (macho) & $6,500 \mathrm{~cm}$ & $5,200 \mathrm{~cm}$ \\
Média & $6,156 \mathrm{~cm}$ & $5,400 \mathrm{~cm}$ \\
Desvio Padrão & $0,688 \mathrm{~cm}$ & $0,400 \mathrm{~cm}$
\end{tabular}

Quadro 2. Valores biométricos do músculo diafragma para animais fêmeas da espécie Sagui-de-tufo-branco (Callithrix jacchus)

\begin{tabular}{ccc}
\hline Animal & Comprimento & Largura \\
\hline N3 (Fêmea) & $5,863 \mathrm{~cm}$ & $5,130 \mathrm{~cm}$ \\
N4 (Fêmea) & $6,800 \mathrm{~cm}$ & $5,700 \mathrm{~cm}$ \\
Média & $6,331 \mathrm{~cm}$ & $5,415 \mathrm{~cm}$ \\
Desvio Padrão & $0,937 \mathrm{~cm}$ & $0,570 \mathrm{~cm}$
\end{tabular}

eixo maior da fibra muscular e consistia num arranjo repetitivo de sarcômeros. Os núcleos se apresentavam ovalados formando aglomerados periféricos nas células. Notou-se a presença das consideradas capsulas musculares já descritas em tratados clássicos de histologia (Banks 1992). Sendo estes: o epimísio e o endomísio, este formado pela lâmina

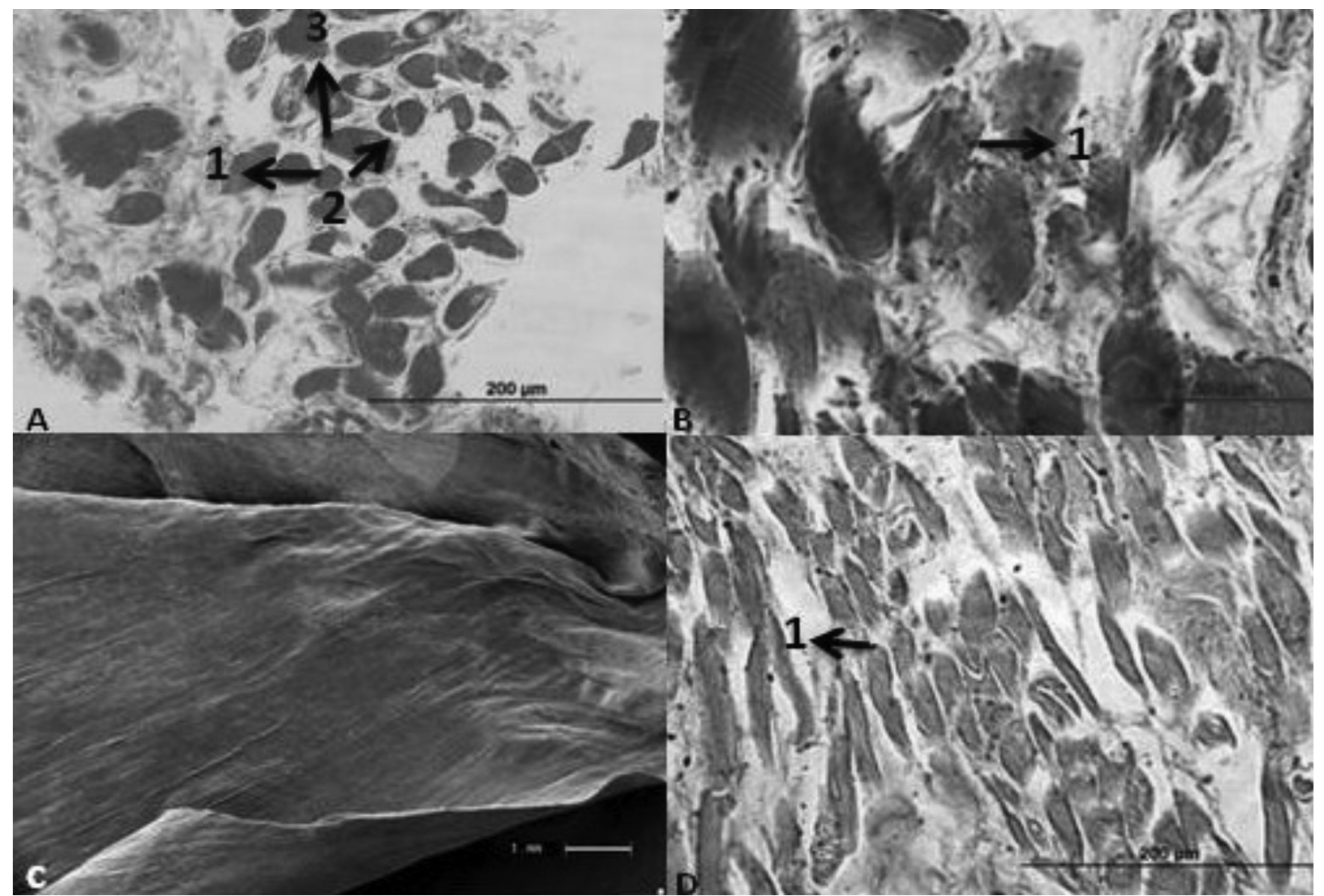

Fig.3. (A) Corte transversal da porção muscular diafragmática. Observa-se o grande diâmetro das fibras musculares descontínuos, constituída por mais de um núcleo (1) o qual está localizado na periferia, endomísio (2) e o epimísio (3). Tricrômio de Masson, 40x. (B) Porção muscular evidenciando os núcleos (1) na periferia das células. Tricrômio de Masson, 100x. (C) A eletromicrografia de varredura mostra a fina espessura do músculo diafragma e as fibras musculares dispostas paralelamente. (D) Corte longitudinal da porção muscular do músculo diafragma evidenciando os núcleos (1) na periferia da fibra muscular. HE, 100x 
basal da fibra muscular, associado a fibras reticulares (Junqueira 2004) (Fig.3C-D).

\section{DISCUSSÃO}

A topografia e estrutura dos diafragmas analisados para a espécie Callithrix jacchus foi de encontro com as descrições presentes na literatura. As quais afirmam que a fixação esquelética do músculo diafragma inicia-se no processo xifóide movendo-se lateralmente para a extremidade ventral das cartilagens costais da sétima à décima terceira costela, transversal ao processo da primeira vértebra lombar. E o centro tendíneo caracteriza-se por ser uma aponeurose central fina, composta por fibras de colágeno entrelaçadas (Harrison 2005).

A respeito dos tipos de colágenos presentes no centro tendineo sabe-se que para as cabras tais fibras são compostas por $58,9 \%$ de fibras do tipo I e por $41,1 \%$ de fibras do tipo II, mas já para os seres humanos, tem-se valores com mais de $50 \%$ para as fibras do tipo I, tendo estas diâmetros idênticos (Ibebunjo 1993).

Diversas foram as descrições morfológicas realizadas para o diafragma em diferentes espécies animais, abrangendo forma, orientação do músculo e tendão, que variavam de acordo com a espécie de enfoque. Em grande parte dos mamíferos já estudados, o centro tendíneo é relativamente fino e transpassado pela veia cava caudal, aorta, ducto torácico, veia ázigos e o esôfago. No cão, por exemplo, o centro tendíneo ocupa aproximadamente $21 \%$ da superfície do diafragma e conseqüentemente $79 \%$ destina-se a porção muscular (Gordon et al. 1989). Havendo uma distribuição das parcelas de força muscular nas aberturas presentes no centro tendíneo de forma biomecânica estável (Hildebrand 1995).

Já com relação a porção muscular do diafragma, em um estudo realizado utilizando-se a espécie Oryctolagus cuniculus (coelho) e Callithrix jacchus (sagui-de-tufo-branco) verificou-se que tal porção era composta por fascículos simétricos, o que corrobora com o presente estudo onde verificou-se também a presença de tais estruturas. No Oryctolagus cuniculus, por exemplo, o diafragma é representado por oito fascículos musculares de forma simétrica em cada antímero do animal (Pompeu et al. 1992).

Tal porção é formada por músculo esquelético o qual consiste em feixes de fibras alongadas com a extremidade ligeiramente afilada ou arrendodada, multinucleadas, tendo núcleos ovais, situados na periferia da célula. Entretanto alguns destes podem estar localizados em região central, decorrente principalmente a artefatos ou por estarem próximos ao sarcolema, acima ou abaixo do plano de observação. Cada fibra contém as miofibrilas, feixes cilíndricos de filamentos, dispostas longitudinalmente em uma matriz de sarcoplasma que se encontra limitada por uma fina membrana, o sarcolema (Banks 1992, Junqueira 2004). Ao redor de cada fibra, externamente ao sarcolema, existe uma fina camada de tecido conjutivo, denominado de endomísio. E cada feixe de fibras se encontra circundado por uma maior quantidade de tecido conjuntivo, chamado de perimisio, e por final a camada externa ao redor de todo o musculo é o epimisio (Carneiro 1992, Getty 2000).
O diafragma da espécie Trichechus manatus latirostris (peixe-boi) diferentemente de outros mamíferos apresenta fibras do centro tendíneo que se anexam as epífises ósseas e se estendem ventralmente nos corpos vertebrais, produzindo assim dois hemidiafragmas. Desta forma localiza-se no plano dorsal e não transversal como encontrado na maioria dos mamíferos, e possuem uma forma alongada de "I" e não de "V" como em outras espécies (Rommel et al. 2000). Sendo tal formato em "V" o encontrado para os espécimes analisados de Callithrix jacchus, ao contrário do observado para a espécie Cavia porcellus (porquinho-da-índia) que deteve um formato de similar a letra "U". mas sendo similar ao encontrado para as espécies Didelphis albiventris (gambá-orelhas-brancas) e para o rato albino (Cassel et al. 2001).

Ainda para o gambá-de-orelhas-brancas este deteve uma área diafragmática média de $54,33 \pm 3,8 \mathrm{~cm}^{2}$, valor este similar ao encontrado para os animais domésticos e de laboratório, indicando assim que o tamanho da área do diafragma é diretamente proporcional ao tamanho do animal analisado (Cassel et al. 2001).

A relação feita entre comprimento e tensão do diafragma em mamíferos (cães, ratos, macacos, gatos e porcos) com a de outros grupos musculares esqueléticos permitiu concluir que por haver uma mistura de tipos de fibras musculares rápidas e fibras musculares lentas nos mesmos não ocorreu resultados diferentes entre o diafragma e os musculos analisados bem como entre as espécies analisadas (Cooper \& Schiller 1975, Sisson \& Grossman 1978, McCully et al. 1983, Dyce et al. 1987).

Segundo English \& Weeks (1987), Loeb et al. (1987), Gaunt \& Gans (1990) e Lessa et al. (2012), o músculo diafragma nos mamíferos apresenta descontinuidade na arquitetura das fibras musculares, sendo esta uma característica importante para garantir o sincronismo da contração muscular, corroborando com os achados histológicos observados neste estudo.

\section{CONCLUSÕES}

As estruturas anatômicas analisadas no diafragma do sagui-de-tufo-branco (Callithrix jacchus) permitiram concluir que não houve diferença morfológica significativa entre machos e fêmeas.

A topografia e a presença de três aberturas (forame da veia cava caudal, hiato aórtico e esofágico) em sua extensão corroboram com descrições na literatura clássica para outros mamíferos.

A presença de um centro tendíneo similar a lera "V" difere do encontrado para animais como o peixe-boi e porquinho-da-india mas é similar ao encontrado para o gambá-de-orelhas-brancas e rato albino.

No que diz respeito aos achados histológicos conclui-se que as fibras musculares estão dispostas de forma organizada, apresentam diâmetro grande e núcleos basais, tendo, portanto, características similares do músculo estriado esquelético tanto nos animais machos como nas fêmeas.

Agradecimentos.- Ao criadouro comercial de animais selvagens, a A.J.B. Soares, pela disponibilidade e fornecimento dos animais analisados e à Médica Veterinária Marta Brito Guimarães. 


\section{REFERÊNCIAS}

Banks W.J. 1992. Sistema Músculo-esquelético, p.289-313. In: Banks W.J. (Ed.), Histologia Veterinária Aplicada. 2ª ed. Ed.Manole, São Paulo.

Cassel F.D., Soares J.C., Torrejais M.M. \& Matheus S.M.M. 2001. Anatomical study of the diaphragm of the opossum (Didelphis albiventris). Anat. Histol. Embryol. 31:132-138.

Cooper G. \& Schiller A.L. 1975. Anatomy of the Guinea Pig. Harvard University Press, Cambridge, p.259-263.

Dyce K.M., Sack W.O. \& Wensing C.J.G. 1987. Textbook of Veterinary Anatomy. W.B. Saunders, Philadelphia. 820p.

English A.W. \& Weeks O.L. 1987. An anatomical and functional analysis of cat biceps femoris and semitendinosus muscle in domestic goats. J. Morphol. 191:161-175.

Gaunt A.S. \& Gans C. 1990. Architecture of chicken muscles: Short fibre patterns and their ontogeny. Proc. R. Soc. B, Biol Sci, 240:351-362.

Getty R. 1986. Anatomia dos Animais Domésticos. Vol.1. 5a ed. Guanabara Koogan, Rio de Janeiro. 1134p.

Gordon D.C., Hammond C.G.M., Fisher J.T. \& Richmond F.J.R. 1989. Muscle-fiber architecture, innervation, and histochemistry in the diaphragm of the cat. J. Morphol. 201:131-143.

Harrison G.R. 2005. The anatomy and physiology of the diaphragm, p.4558. In: Braunwald E., Hauser S.L., Longo D.L., Fauci A.S., Kasper D.L., Wiener C.M., Nivatpumin P.J., Brown C.D. \& Hemnes A.R. (Eds), Princípios de Medicina Interna. 16 $\underline{\text { a }}$ ed. McGraw-Hill, Rio de Janeiro.

Hildebrand M. 1995. Reproductive system and urogenital ducts, p.299315. In: Ibid. (Ed.), Analysis of Vertebrate Structure. $4^{\text {th }}$ ed., John Wiley and Sons Inc., New York.
Ibebunjo C. 1993. Type, diameter and distribution of fibres in some respiratory and abdominal muscles of the goat. Vet. Res. Commun. 17:171182.

Junqueira L.C. \& Carneiro J. 2004. Tecido muscular, p.184-205. In: Junqueira L.C. \& Carneiro J. (Eds), Histologia Básica. 10ª ed. Guanabara Koogan, Rio de Janeiro.

Lessa T.B., Silva L.C.S., Constantino M.V.P., Santos P.R.S., Assis-Neto A.C., Bombonato P.P. \& Ambrósio C.E. 2012. Morfologia comparativa do diafragma do sagui-de-tufo-branco e do sagui-de-cara-branca. Biotemas 25(1):119-124.

Loeb G.E., Pratt C.A. \& Chanaud C.M. 1987. Distribution and innervation of short interdigitated muscle fibers in parallelfibered muscles of the cat hindlimb. J. Morphol. 191:1-15.

Mansfield K. 2003. Marmosets models commonly used in biomedical research. Comp. Med. 53(4):383-392.

McCully K.K. \& Faulkner J.A. 1983. Length-tension relationship of mammalian diaphragm muscles. J. Appl. Physiol. 54(6):1681-1686.

Pinto Filho S.T.L., Brondani J.T., Graça D.L. \& Schossler J.E. 2003. Restauração do diafragma de felino com enxerto autólogo de pericárdio. Acta Cirur. Bras. 18(5):471-477.

Pompeu E., Liberti E.A., Osaka J.T., Rodrigues Jr A.J. \& Tolosa E.M.C. 1992. The rabbit's diaphragm innervation. Revta Bras. Ciênc. Morfol. 1:3336.

Rommel S. \& Reynolds J.E. 2000. Diaphragm structure and function in the Florida Manatee (Trichechus manatus latirostris). Anat. Rec. 259:41-51.

Sisson S. \& Grossman J.D. 1978. Anatomia de los Animales Domesticos. $4^{\underline{a}}$ ed. Salvat, Barcelona. 1416p. 